ARTICLE

\title{
Giant anomalous Hall effect from spin-chirality scattering in a chiral magnet
}

Yukako Fujishiro (10) 1,6凶 , Naoya Kanazawa (1) 1,6凶, Ryosuke Kurihara², Hiroaki Ishizuka', Tomohiro Hori (1) 1, Fehmi Sami Yasin (10 3, Xiuzhen Yu (1) 3, Atsushi Tsukazaki (1) 4, Masakazu Ichikawa', Masashi Kawasaki 1,3, Naoto Nagaosa ${ }^{1,3}$, Masashi Tokunaga (i) ${ }^{2}$ \& Yoshinori Tokura (i) 1,3,5凶

The electrical Hall effect can be significantly enhanced through the interplay of the conduction electrons with magnetism, which is known as the anomalous Hall effect (AHE). Whereas the mechanism related to band topology has been intensively studied towards energy efficient electronics, those related to electron scattering have received limited attention. Here we report the observation of giant AHE of electron-scattering origin in a chiral magnet $\mathrm{MnGe}$ thin film. The Hall conductivity and Hall angle, respectively, reach 40,000 $\Omega^{-1}$ $\mathrm{cm}^{-1}$ and $18 \%$ in the ferromagnetic region, exceeding the conventional limits of AHE of intrinsic and extrinsic origins, respectively. A possible origin of the large AHE is attributed to a new type of skew-scattering via thermally excited spin-clusters with scalar spin chirality, which is corroborated by the temperature-magnetic-field profile of the AHE being sensitive to the film-thickness or magneto-crystalline anisotropy. Our results may open up a new platform to explore giant AHE responses in various systems, including frustrated magnets and thin-film heterostructures.

\footnotetext{
${ }^{1}$ Department of Applied Physics, The University of Tokyo, Bunkyo-ku, Tokyo 113-8656, Japan. ${ }^{2}$ The Institute for Solid State Physics (ISSP), The University of Tokyo, Kashiwa, Chiba 277-8581, Japan. ${ }^{3}$ RIKEN Center for Emergent Matter Science (CEMS), Wako, Saitama 351-0198, Japan. ${ }^{4}$ Institute for Materials Research (IMR), Tohoku University, Aoba-ku, Sendai 980-8577, Japan. ${ }^{5}$ Tokyo College, The University of Tokyo, Bunkyo-ku, Tokyo 113-8656, Japan. ${ }^{6}$ These

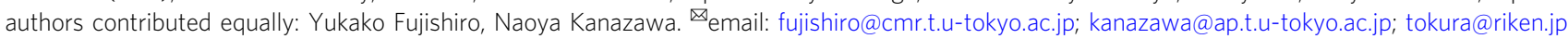


T he quantum nature of electrons leads to rich electromagnetic responses in correlated electron systems, in particular, through the interaction with magnetism ${ }^{1}$. The anomalous Hall effect (AHE) is one such phenomenon, where the Hall effect occurs usually as a consequence of magnetic-ordering and spin-orbit coupling (SOC) ${ }^{2}$. This phenomenon potentially benefits applications as well, since a large Hall response provides a pathway to energy efficient electronic or spintronic devices through the suppression of the longitudinal current which entails the Joule heating. To date, studies aiming at a large Hall response have focused on the gauge field arising from the electronic bands and magnetic orders. For example, a small magnetization can produce a large net Berry curvature in topological semimetals ${ }^{3-7}$. The extreme example is the quantized AHE in magnetic topological insulators, which realizes the infinite Hall angle and hence dissipation-less conduction ${ }^{8,9}$. Another direction is to tailor a non-coplanar magnetic order with a geometrical correlation as characterized by scalar spin chirality (SSC) $\chi=\mathbf{S}_{i} \cdot\left(\mathbf{S}_{j} \times \mathbf{S}_{k}\right)$, which results in a large net Berry curvature through the coupling between conduction electrons ${ }^{10-13}$. Here, $\mathbf{S}_{i}, \mathbf{S}_{j}$, and $\mathbf{S}_{k}$ represent spins at three neighboring atomic sites $(i, j, k)$. In particular, a dense topological spin texture with a short magnetic period leads to large topological Hall effect (THE) ${ }^{14,15}$. However, the Hall conductivity of these "intrinsic" mechanisms has the upper threshold set by the Berry curvature. In the case of momentum space Berry curvature, the consequent Hall conductivity should be less than $e^{2} / h a$ ( $h$ and $a$ being Planck's constant and a typical lattice constant values), and hence of the order of $\sigma_{x y}=10^{2}-10^{3}$ $\Omega^{-1} \mathrm{~cm}^{-1}$.

On the other hand, the contribution of electron scattering (conventionally termed "extrinsic" mechanism) is not restricted by the Berry curvature. Nevertheless, extrinsic mechanisms have rarely been studied in the context of large AHE responses, due to its small Hall conductivity $\left(\sigma_{x y}\right)$. The only exception occurs in the extremely high conductivity regimes (empirically above $\sigma_{x x}>5 \times 10^{5} \Omega^{-1} \mathrm{~cm}^{-1}$ ), where the skew-scattering (asymmetric electron scattering due to the SOC at impurities) dominates $\sigma_{x y}$ with a characteristic scaling relation $\left(\sigma_{x y} \propto \sigma_{x x}\right)^{16,17}$. Even in that case, the Hall angle $\left[\Theta_{\mathrm{H}}=\tan ^{-1}\left(\sigma_{x y} / \sigma_{x x}\right)\right]$ remains a constant value, i.e., $\sigma_{x y} \propto \sigma_{x x}$, which is as small as $0.1-1 \%$ because the spin-orbit interaction is usually smaller than the width of the virtual bound state and also skew-scattering requires the phase shifts of different orbital angular momenta ${ }^{16-21}$.

\section{Results}

MnGe thin film with topological spin texture. Here we report the observation of a giant AHE in a chiral magnet MnGe thin film, where the giant Hall angle (18\%) leads to a Hall conductivity reaching $\sigma_{x y} \sim 40,000 \Omega^{-1} \mathrm{~cm}^{-1}$, being two orders of magnitude larger than the intrinsic AHE, even with a moderate longitudinal conductivity $\left(\sigma_{x x} \sim 2 \times 10^{5} \Omega^{-1} \mathrm{~cm}^{-1}\right)$. The target material is the epitaxially grown thin films of $\mathrm{MnGe}^{22}$, a member of the B20type chiral magnets hosting topological spin textures ${ }^{23,24}$. The crystal structure belongs to the non-centrosymmetric space group $P 2_{1} 3$, where the lattice chirality is characterized by the stacking direction of the atoms as viewed from [111] axis (only one enantiomeric form is shown in Fig. 1a). Since the lack of inversion symmetry allows Dzyaloshinskii-Moriya interaction (DMI), twisted spin structures such as helical or non-coplanar structures are often observed in B20-type magnets ${ }^{23,24}$. Among them, $\mathrm{MnGe}$ hosts unique magnetic textures composed of spin hedgehogs and anti-hedgehogs. In a bulk sample, those magnetic defects form a short-period lattice $(\sim 2.8 \mathrm{~nm})$ with large magnetic fluctuations even persisting in the nominally ferromagnetic (FM) region ${ }^{24}$. The hedgehog lattice is deformed to a rhombohedral form
(Supplementary Fig. 1$)^{22}$ or decomposed into multi-domains with differently oriented helical structures ${ }^{25}$ in strained thin films (see Supplementary Note 1 for comment on the magnetic texture in thin films). In either case, there exist chains of hedgehogs and anti-hedgehogs connected by skyrmion-strings. Such a noncoplanar magnetic order produces a THE in metallic systems including MnGe $\left(\sigma_{x x}=2.0 \times 10^{5} \Omega^{-1} \mathrm{~cm}^{-1}\right.$ at $2 \mathrm{~K}$ ) (Fig. $1 \mathrm{~b}$ ); the large negative THE below $T<50 \mathrm{~K}$ (Fig. 1c) is ascribed to emergent magnetic field from the skyrmion-strings (inset in Fig. 1c) $)^{14}$.

Hall conductivity in the ferromagnetic region. The main focus of this work is on the high-magnetic-field Hall response shown in Fig. 1d, where $\sigma_{x y}$ shows a striking enhancement reaching $\sim 40,000 \Omega^{-1} \mathrm{~cm}^{-1}$ at the temperature $T=2 \mathrm{~K}$, with a sharp peak structure at around the magnetic field $B=14 \mathrm{~T}$ (see Supplementary Fig. 2 for the resistivity data). Whereas $\sigma_{x x}=2.02 \times 10^{5} \Omega^{-1} \mathrm{~cm}^{-1}$ at $2 \mathrm{~K}$ (Fig. 1b) belongs to the empirical intrinsic regime where the Berry curvature mechanism is dominant, the observed $\sigma_{x y}$ far exceeds the threshold value of the intrinsic AHE from the momentum space, which is roughly estimated from the quantization limit $\left(\sim e^{2} / h a=800 \Omega^{-1} \mathrm{~cm}^{-1}\right.$ for $\mathrm{MnGe}$ ) in three-dimensions (denoted by the dashed line in Fig. 1d $)^{17}$. Here, $a=4.795 \AA$ for MnGe. Upon increasing the temperature, $\sigma_{x y}$ is rapidly suppressed while showing a broader peak structure. Above $T \sim 70 \mathrm{~K}, \sigma_{x y}$ follows the conventional behavior of the intrinsic AHE, which scales with the magnetization $^{2}$ (Supplementary Fig. 2).

Scaling relation and temperature-magnetic-field profile of the Hall response. To understand the characteristics of this large Hall response observed at low temperatures, we first plotted the data of $\sigma_{x y}$ (Fig. 1d) against $\sigma_{x x}$ (Supplementary Fig. 2) to investigate their scaling relation, which has been typically used to identify the mechanism of the $\mathrm{AHE}^{2,16,17}$. Figure 2a shows the plot for various samples with different film-thicknesses $(t=80,160$, and $300 \mathrm{~nm}$ ) for $T=2-30 \mathrm{~K}$ (see Supplementary Fig. 3 for the complete data set). The displayed data points $\left(\sigma_{x x}, \sigma_{x y}\right)$ are those exhibiting the maximum Hall angle at each temperature. In every sample, the scaling relation between $\sigma_{x y}$ and $\sigma_{x x}$ is linear $\left(\sigma_{x y} \propto \sigma_{x x}\right)$, which is a typical feature expected for skewscattering mechanism (see Supplementary Fig. 4 and Supplementary Note 4 for detailed discussions). To overview this Hall response in the $B-T$ plane, the contour map of $\sigma_{x y}$ is shown with the magnetic phase diagram in Fig. $2 \mathrm{~b}$. The striking enhancement of the Hall response appears at low temperatures below $50 \mathrm{~K}$, in proximity to the FM phase boundary. The constant- $B$ cut of $\sigma_{x y}$ shows a peak structure at finite temperature, and the peak structure shifts to higher temperatures under higher magnetic fields (Fig. 2c).

The above features of the skew-scattering in MnGe are distinct from the conventional skew-scattering induced by the nonmagnetic chemical defects or single-spin impurity $2,16-21$, while showing the similar linear $\left(\sigma_{x y} \propto \sigma_{x x}\right)$ scaling relation. In the conventional skew-scattering, the perturbatively small SOC compared to the electron bandwidth results in a small Hall angle of $0.1-1 \%$. Also, $\sigma_{x y}$ should be monotonously increased to lower temperature with higher $\sigma_{x x}$. Hence, not only the giant Hall angle of $18 \sim 22 \%$ (Fig. 2a), but also the non-monotonous $B-T$ profile (Fig. 2b, c) are both beyond the conventional understanding of skew-scattering. One possibility for this unconventional skewscattering is the recently proposed "spin-chirality skew-scattering" mechanism ${ }^{26}$, where the thermally fluctuating spins in the 

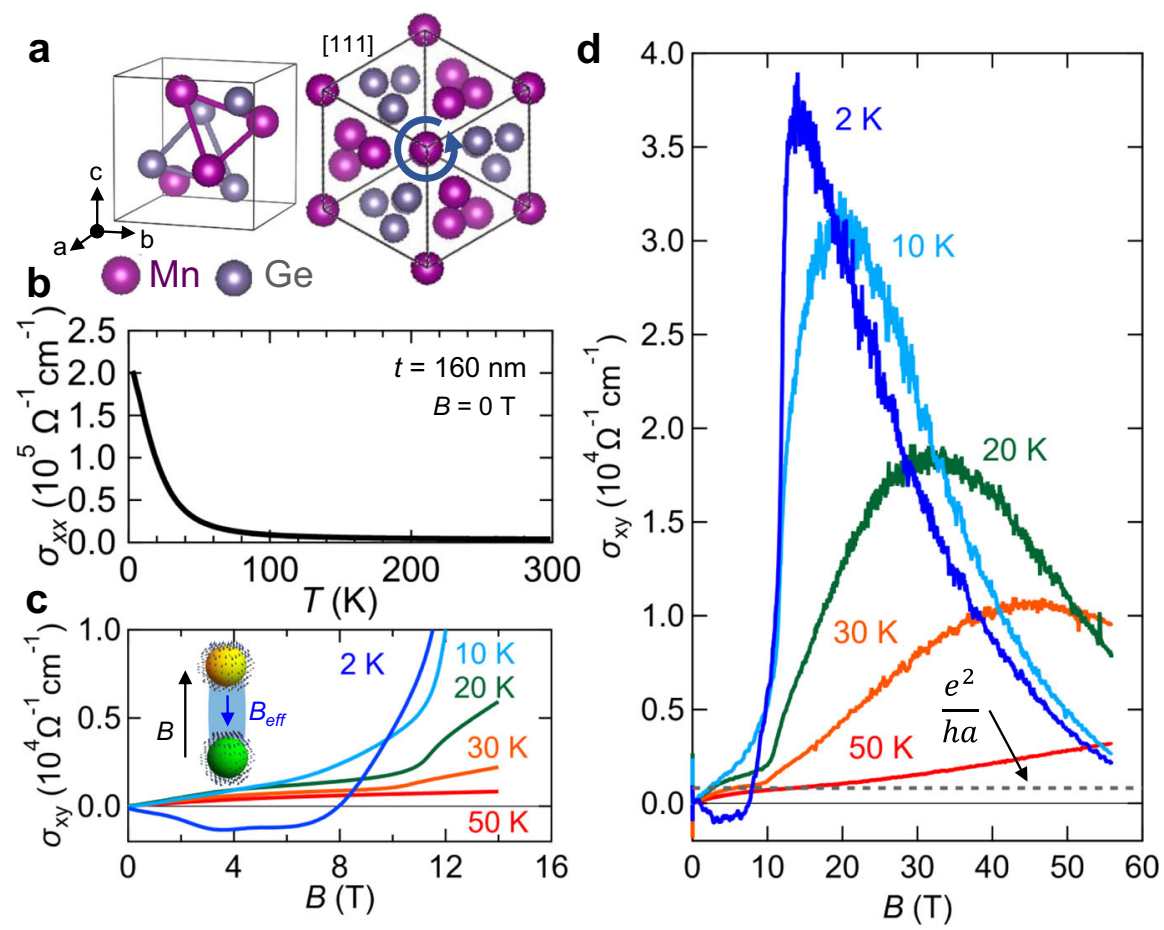

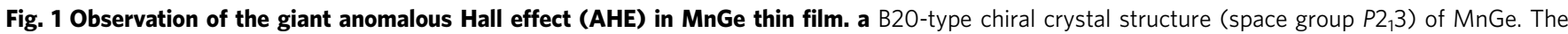
chirality is characterized by the stacking direction of atoms as viewed from [111] direction. $\mathbf{b}$ Temperature dependence of conductivity $\left(\sigma_{x x}\right)$ at zero magnetic field for the film-thickness of $t=160 \mathrm{~nm}$. c Magnetic-field dependence of Hall conductivity $\left(\sigma_{x y}\right)$ at various temperatures for $t=160 \mathrm{~nm}$. The negative dip structures are attributed to topological Hall effect arising from the formation of spin hedgehogs and anti-hedgehogs bridged by skyrmionstrings (shown in the inset). d High-magnetic-field data of $\sigma_{x y}$ at various temperatures for $t=160 \mathrm{~nm}$, showing a large enhancement. The value of the quantization limit $\left(e^{2} / h a\right)$ in three-dimensions is denoted by a dashed line. The observed giant AHE far exceeds the value allowed by the intrinsic AHE.
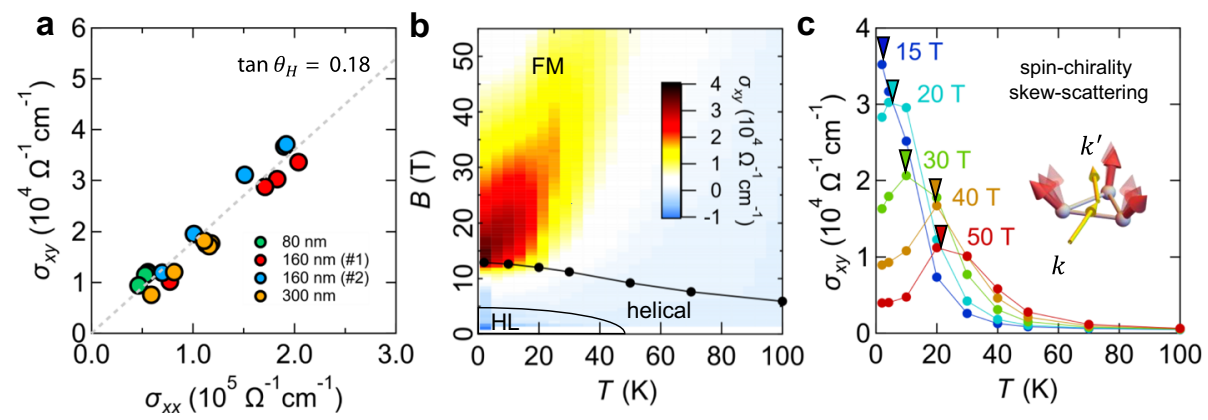

Fig. 2 Characteristics of the AHE observed in MnGe thin film, suggesting a spin-chirality skew-scattering. a Plot of Hall conductivity $\left(\sigma_{x y}\right)$ versus conductivity $\left(\sigma_{x x}\right)$ for the thickness of $t=80,160$, and $300 \mathrm{~nm}$. The data are taken from the peak positions of the Hall angle below $30 \mathrm{~K}$. The linear relation $\left(\sigma_{x y} \propto \sigma_{x x}\right)$ appears in a certain $B-T$ region with a large Hall angle of $\sim 18 \%$. b Contour plot of $\sigma_{x y}$ in the $B-T$ space with the magnetic phase diagram consisting of the hedgehog lattice $(\mathrm{HL})$, helical, and the ferromagnetic (FM) phases. c Constant- $B$ cut of $\sigma_{x y}$ showing a peak structure at a finite temperature. The triangles denote the peak positions, which shifts to a higher temperature at a higher magnetic field. These results are suggestive of the relation to the thermal excitation of the scalar spin chirality (inset in Fig. 2c).

FM state act as the spin-clusters with the SSC (inset in Fig. 2c). Hence, the magnetic scattering contributes to the AHE. In principle, the scattering process there does not involve SOC and the Hall angle is predicted to be significantly large compared to the conventional skew-scattering. In particular, the Hall angle in the order of $10 \%$ is anticipated to show up when the size of the spin-cluster is comparable to the inverse of the Fermi-wavevector $\left(\sim \frac{1}{k_{\mathrm{F}}}\right)$, which corresponds to the resonance condition of the interference effect ${ }^{26}$. Moreover, the observed $B-T$ profile of the AHE is also consistent with the spin-cluster scenario. Under fixed magnetic field, the spin-cluster AHE is expected to be maximized at a finite temperature as shown in Fig. 2c. Two types of spin excitations with opposite SSC are thermally excited, being responsible for skew-scatterings with opposite Hall angles. Because those SSC excitations have different activation energies due to the presence of DMI, the cancellation of AHE signal only occurs above the temperature where the SSC excitations with the higher activation energy start to proliferate. Although the temperature dependence of the Hall signal under fixed magnetic field is consistent with our analytical calculations and Monte Carlo simulations (see Supplementary Figs. 5 and 6, and Supplementary Notes 5 and 6 for details), the reason why the Hall signal is enhanced at high magnetic field under fixed temperature remains elusive and needs further theoretical investigations. This may be attributed to the divergent spin correlation and the criticality around the FM transition. The 

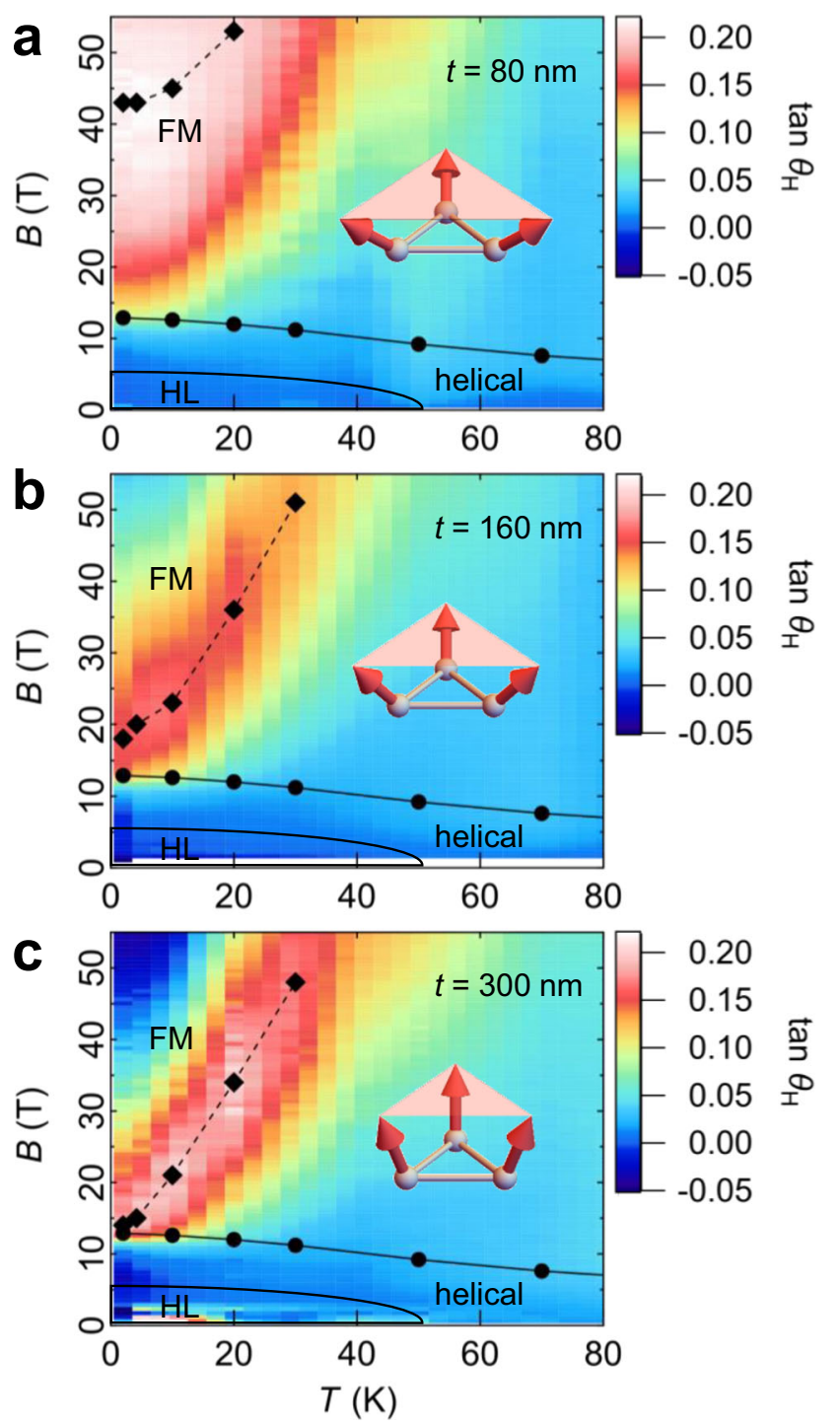

Fig. 3 Film-thickness dependence of the temperature-magnetic-field profile of the AHE in MnGe thin film. Contour plot of $\tan \Theta_{H}\left(=\sigma_{x y} / \sigma_{x x}\right)$ for the thickness of $t=80$ (a), 160 (b), and $300 \mathrm{~nm}$ (c). The solid diamond markers connected by a dashed line represent the maximum point of $\tan \theta_{\mathrm{H}}$ at each temperature. The insets are the intuitive schematics to explain the fact that the larger scalar spin chirality excitation is more favorable in thinner films with enhanced in-plane magnetic anisotropy.

former invalidates the short-correlation-length assumption used in the skew-scattering theory, while the latter may affect the transport phenomena in a nontrivial way. Study on such effects is interesting but requires intensive theoretical investigations that go beyond the scope of this paper.

Film-thickness dependence of the Hall response. To elaborate on the possibility of the spin-cluster mechanism, we have controlled the magneto-crystalline anisotropy by changing the filmthickness; the SANS experiment on MnGe thin films has revealed the enhanced in-plane magnetic anisotropy with decreasing filmthickness $^{22}$ (Supplementary Fig. 1). Therefore, the spins can "tilt" easier from the field-polarized direction in the FM state in thinner films and we expect that the SSC excitation in the collinear spin background has a corresponding lower energy. In other words, larger SSC can be produced due to the enhanced in-plane anisotropy in the thinner films as schematically illustrated in the insets of Fig. 3. We note that the insets of Fig. 3 are overdrawn to emphasize the difference induced by the film-thickness. The $B-T$ profile of the AHE shows a clear variation with film-thickness of $t=80,160$, and $300 \mathrm{~nm}$ (Fig. 3, see also Supplementary Figs. 2 and 3 for the complete data set). While the maximum value of the Hall angle is almost independent of the film-thickness, the $B-T$ profile of the AHE changes dramatically as shown in Fig. 3. In thinner films with enhanced in-plane anisotropy, a large Hall angle shows up at lower temperature and higher applied magnetic field, where the SSC excitation costs more energy. We also provide analytical calculations on the effect of easy-plane anisotropy in Supplementary Note 5.

Comparison with other materials. The discovery of this new type of the AHE, showing giant Hall conductivity and Hall angle simultaneously, provides a distinct exception in the universal scaling curve of $\sigma_{x y}$ versus $\sigma_{x x}$ established for various ferromagnets 2,17 (Fig. 4). The Hall angle of $18 \%$ in MnGe gives one- or two-orders of magnitude larger $\sigma_{x y}$ for a given $\sigma_{x x}$, compared to the conventional skew-scattering, resulting in an upward shift of the scaling plot with a linear relation $\left(\sigma_{x y} \propto \sigma_{x x}\right)$. We also emphasize that $\sigma_{x y}$ from the intrinsic AHE cannot reach this regime, due to the limitations set by the Berry curvature in momentum space. Recently, the spin-cluster AHE was also reported in a frustrated magnet $\mathrm{KV}_{3} \mathrm{Sb}_{5}$, with large Hall conductivity of $15,507 \Omega^{-1} \mathrm{~cm}^{-1}$ and Hall angle of $1.8 \%{ }^{27}$. We assume that the difference in the Hall angle between MnGe and $\mathrm{KV}_{3} \mathrm{Sb}_{5}$ may be related to the resonance condition for the spinchirality skew-scattering. For MnGe, the unusually short magnetic period $(\lambda \sim 2.8 \mathrm{~nm})$ has been discussed in terms of the conduction-electron mediated mechanisms ${ }^{24,28,29}$ such as the Rudermann-Kittel-Kasuya-Yosida (RKKY) interaction; this can automatically tune the size of the spin cluster to the typical size of $\sim \frac{1}{k_{\mathrm{F}}}$, satisfying the resonance condition. Hence the RKKY magnets or the metallic spin-glass systems would be the promising candidates for achieving a large Hall angle. However, the true nature of the excitation modes for the SSC in MnGe remains to be a challenge for future study. For instance, the origin of the SSC can be either the slightly tilted spin clusters or the pairwise excitations of spin hedgehogs and anti-hedgehogs connected by the skyrmion-strings. We speculate that the latter may be the case, given the fact that the magnetic ground state of MnGe at low temperature $(T<50 \mathrm{~K})$ is an ordered state of these spin singularities.

\section{Discussions}

Although we have focused on the three-spin correlation with SSC, the two-spin correlation with vector spin chirality can also produce the AHE. In addition to the intrinsic AHE induced by the two-spin correlation $^{30,31}$, the extrinsic AHE could also emerge when combined with the SOC as long as the Bloch states host finite Berry curvature $^{32}$. If the SOC played the dominant contribution, the AHE in the high field limit would be large, while the experiment shows the rapid quench of $\sigma_{x y}$ as shown in Fig. 1d. Therefore, the SSC is most important, and the three-spin correlation without SOC should dominate over the two-spin correlation with SOC.

One another possibility for the large Hall response observed in MnGe films is the emergence of high-mobility carriers in the FM region, which can result in $\sigma_{x y}$ with a sharp peak structure, as typically observed in Dirac or Weyl semimetals ${ }^{33}$. We found that the observed Hall conductivity could be roughly reproduced by two-carrier Drude model, by assuming the presence of high-mobility $\left(\sim 690 \mathrm{~cm}^{2} \mathrm{~V}^{-1} \mathrm{~cm}^{-1}\right)$ and low-carrier-density $\left(\sim 9.4 \times 10^{20} \mathrm{~cm}^{-3}\right)$ hole pocket (see Supplementary Note 7 and 


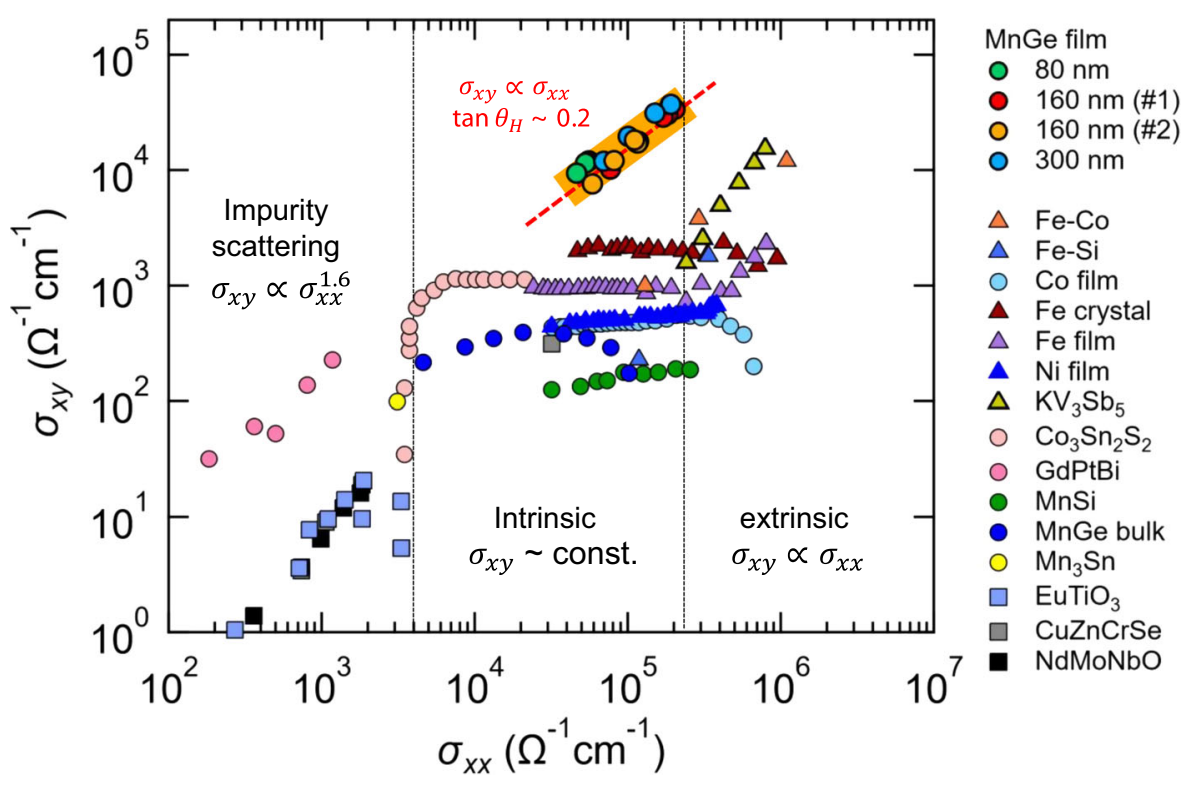

Fig. 4 Full logarithmic plot of $\sigma_{\mathbf{x y}}$ versus $\boldsymbol{\sigma}_{\mathbf{x x}}$ for various materials. The shaded region with a red dashed line indicates a linear relation $\left(\sigma_{x y} \propto \sigma_{x x}\right)$ in MnGe thin films with a giant Hall angle reaching $18 \sim 22 \%$ for the thickness of $t=80,160$, and $300 \mathrm{~nm}$. The data are taken from the peak positions of the Hall angle below $30 \mathrm{~K}$. The large Hall conductivity and the Hall angle realized in $\mathrm{MnGe}$ thin films result in a clear deviation from the conventional scaling plots established for ferromagnets $2,16,17$. The reported data for other materials are cited from literature 6,7,14,16,17,20,27,38-40.

Supplementary Figs. 7-9 for detailed discussions). However, this assumption that the observed Hall response is dominated by the normal Hall effect is less plausible, partly because the giant Hall response is not observed in bulk polycrystalline $\mathrm{MnGe}$ which has almost the same $\sigma_{x x}\left(1.6 \times 10^{5} \Omega^{-1} \mathrm{~cm}^{-1}\right.$ at $\left.2 \mathrm{~K}\right)$ with that of the thin film, but the SSC excitation effect may be canceled out due to the randomly oriented crystalline domains (see Supplementary Note 8 and Supplementary Fig. 10 for detailed discussions). Further experiments such as the direct observation of electronic structure, especially in the FM region, would be necessary to discuss the possibility of large normal Hall effect or the presence of magnetic Weyl points in MnGe. Moreover, the characteristic features discussed in this work, such as the scaling relations, nonmonotonous $B-T$ profile, and magneto-crystalline anisotropy dependence of the Hall response, strongly suggest the spinchirality skew-scattering mechanism argued above.

In conclusion, we have observed giant AHE in the moderately conductive MnGe thin films, which suggests the skew-scattering from the SSC excitation. Since most of the experimentally available materials belong to the intrinsic regime $\left(10^{3}<\sigma_{x x}<10^{5}\right)$ or the dirty regime $\left(\sigma_{x x}<10^{3}\right)$, spin-chirality skew-scattering would provide an opportunity to explore giant AHE responses in various materials. It is expected to show up in systems where the finite thermal average of the SSC is induced by the short-range spin correlation, for instance, by the geometrical frustration or the inversion-symmetry breaking at the interface. In that sense, the large family of frustrated magnets ${ }^{34}$ with triangular, Kagome, and pyrochlore lattices, as well as the heterostructures with interfacial $\mathrm{DMI}^{35}$ would be prospective platforms to explore this new AHE. Unlike the conventional intrinsic AHE which has been limited to ferromagnets or the ordered phases of the SSC, the proposed new paradigm of spin-chirality skew-scattering would be applicable to a wide range of materials; since it is expected even in materials with no magnetic-ordering as well as in the temperature-magnetic-field region where the SSC loses its longrange order. Exploration of giant AHE responses, from a perspective of short-range spin correlation, would open up a new frontier in the discovery of novel electronic functionalities.

\section{Methods}

Thin-film growth. MnGe thin films were grown epitaxially on $\mathrm{Si}(111)$ substrates by the molecular beam epitaxy method ${ }^{22}$. We employed a $2 \mathrm{~nm}$ thick $\mathrm{MnSi}(111)$ buffer layer, which was grown by reacting a deposited Mn layer with the Si(111)$(7 \times 7)$ surface at $250^{\circ} \mathrm{C}$. Then, $\mathrm{Mn}$ and Ge were co-evaporated at approximately $90^{\circ} \mathrm{C}$, followed by annealing at $250^{\circ} \mathrm{C}$. The growth of B20-type MnGe was confirmed by $\theta-2 \theta$ x-ray diffraction scans.

Electric transport measurement. Resistivities up to $14 \mathrm{~T}$ were measured with a conventional four-probe method using a DC option of Physical Properties Measurement System (PPMS, Quantum Design). High-magnetic-field transport measurements up to $56 \mathrm{~T}$ were performed utilizing non-destructive pulse magnets energized by capacitor banks installed at the International MegaGauss Science Laboratory of Institute for Solid State Physics (ISSP), University of Tokyo. The magnetic field was applied perpendicular to the film plane, i.e., (111) plane, while the electric field was applied parallel to $[1 \overline{1} 0]$ direction. The longitudinal conductivity $\left(\sigma_{x x}\right)$ and the Hall conductivity $\left(\sigma_{x y}\right)$ were calculated as $\sigma_{x x}=$ $\rho_{x x} /\left(\rho_{x x}^{2}+\rho_{y x}^{2}\right)$ and $\sigma_{x y}=\rho_{y x} /\left(\rho_{x x}^{2}+\rho_{y x}^{2}\right)$. Here, $\rho_{x x}$ and $\rho_{y x}$ are the longitudinal and Hall resistivity, respectively. We could not perform the transport measurements in thick samples (film-thickness larger than $400 \mathrm{~nm}$ ) due to the formation of microcracks upon cooling.

Dark-field transmission electron microscopy measurement. The epitaxially grown MBE thin films of MnGe are composed of a mixed-domain state of enantiomers, although the different enantiomer (lattice chirality) with an opposite-sign DMI should show an identical contribution to any kind of Hall effect. The size of the chiral domain in MnGe thin films was investigated by the dark-field transmission electron microscopy (TEM) measurement ${ }^{36,37}$. First, we prepared the sample by grinding the $\mathrm{Si}$ substrate down to $\sim 6-8 \mu \mathrm{m}$ using the multiprep and dimple grinding systems. We then utilized precision ion milling (Pips) to remove the remaining Si substrate, leaving the 80,160 , and $300 \mathrm{~nm}$ thick films, which were mounted onto TEM sample holderready Mo annuli. We characterized the orientation of each film using electron diffraction. As in Ref. ${ }^{22}$, we found the [111] axis incidence using selected area electron diffraction within a JEOL JEM 2100 TEM as shown in Supplementary Fig. 10a. We utilized a LN-holder (Gatan 636) for its high angle tilt capability. As shown in Supplementary Fig. 10a, b, the diffraction peaks corresponding to the two domains of interest (labeled A and B in Supplementary Fig. 10b) emerge when the crystal is tilted around the $[11 \overline{2}]$ axis by approximately $22^{\circ}$. The spots were isolated using the objective aperture, and the passing electrons were focused into an image of the domains onto the charge-coupled device (CCD) camera installed within the microscope.

\section{Data availability}

The data sets generated during and/or analyzed during the current study are available from the corresponding author on reasonable request. 
Received: 29 August 2020; Accepted: 30 November 2020;

Published online: 12 January 2021

\section{References}

1. Tokura, Y., Kawasaki, M. \& Nagaosa, N. Emergent functions of quantum materials. Nat. Phys. 13, 1056-1068 (2017)

2. Nagaosa, N., Sinova, J., Onoda, S., MacDonald, A. H. \& Ong, N. P. Anomalous Hall effect. Rev. Mod. Phys. 82, 1539 (2010).

3. Karplus, R. \& Luttinger, J. M. Hall effects in ferromagnets. Phys. Rev. 95, 1154 (1954).

4. Xiao, D., Chang, M.-C. \& Niu, Q. Berry phase effects on electronic properties. Rev. Mod. Phys. 82, 1959 (2010).

5. Fang, Z. et al. The anomalous Hall effect and magnetic monopoles in momentum space. Science 302, 92-95 (2003).

6. Suzuki, T. et al. Large anomalous Hall effect in a half-Heusler antiferromagnet. Nat. Phys. 12, 1119-1123 (2016)

7. Liu, E. et al. Giant anomalous Hall effect in a ferromagnetic Kagomé-lattice semimetal. Nat. Phys. 14, 1125-1131 (2018).

8. Chang, C.-Z. et al. Experimental observation of the quantum anomalous Hall effect in a magnetic topological insulator. Science 340, 167-170 (2013).

9. Tokura, Y., Yasuda, K. \& Tsukazaki, A. Magnetic topological insulators. Nat. Rev. Phys. 1, 126 (2019).

10. Matl, P. et al. Hall effect of the colossal magnetoresistance manganite $\mathrm{La}_{1-x} \mathrm{Ca}_{x} \mathrm{MnO}_{3}$. Phys. Rev. B 57, 10248 (1998).

11. Ye, J. et al. Berry phase theory of the anomalous Hall effect: application to colossal magnetoresistance manganites. Phys. Rev. Lett. 83, 3737 (1999).

12. Taguchi, Y., Oohara, Y., Yoshizawa, H., Nagaosa, N. \& Tokura, Y. Spin chirality, Berry phase, and anomalous Hall effect in a frustrated ferromagnet. Science 291, 2573-2576 (2001).

13. Neubauer, A. et al. Topological Hall effect in the A phase of MnSi. Phys. Rev. Lett. 110, 209902 (2013).

14. Kanazawa, N. et al. Large topological Hall effect in a short-period helimagnet MnGe. Phys. Rev. Lett. 106, 156603 (2011)

15. Kurumaji, T. et al. Skyrmion lattice with a giant topological Hall effect in a frustrated triangular-lattice magnet. Science 365, 914-918 (2019).

16. Miyasato, T. et al. Crossover behavior of the anomalous Hall effect and anomalous Nernst effect in itinerant ferromagnets. Phys. Rev. Lett. 99, 086602 (2007).

17. Onoda, S., Sugimoto, N. \& Nagaosa, N. Quantum transport theory of anomalous electric, thermoelectric, and thermal Hall effects in ferromagnets. Phys. Rev. B 77, 165103 (2008).

18. Smit, J. The spontaneous Hall effect in ferromagnetics II. Physica 24, 39-51 (1958).

19. Berger, L. Side-jump mechanism for the Hall effect of ferromagnets. Phys. Rev. B 2, 4559 (1970)

20. Shiomi, Y., Onose, Y. \& Tokura, Y. Extrinsic anomalous Hall effect in charge and heat transport in pure iron $\mathrm{Fe}_{0.997} \mathrm{Si}_{0.003}$, and $\mathrm{Fe}_{0.97} \mathrm{Co}_{0.03}$. Phys. Rev. B 79, 100404(R) (2009).

21. Hou, D. et al. Multivariable scaling for the anomalous Hall effect. Phys. Rev. Lett. 114, 217203 (2015).

22. Kanazawa, N. et al. Topological spin-hedgehog crystals of a chiral magnet as engineered with magnetic anisotropy. Phys. Rev. B 96, 220414(R) (2017).

23. Bauer, A. \& Pfleiderer, C. Generic aspects of skyrmion lattices in chiral magnets. Springer Ser. Mater. Sci. 228, 1-28 (2016).

24. Fujishiro, Y., Kanazawa, N. \& Tokura, Y. Engineering skyrmions and emergent monopoles in topological spin crystals. Appl. Phys. Lett. 116, 090501 (2020).

25. Repicky, J. et al. Atomic scale visualization of topological spin textures in the chiral magnet MnGe. Preprint at https://arxiv.org/abs/2008.00886 (2020).

26. Ishizuka, H. \& Nagaosa, N. Theory of giant skew scattering by spin cluster. Preprint at https://arxiv.org/abs/1906.06501 (2020).

27. Yang, S.-Y. et al. Giant, unconventional anomalous Hall effect in the metallic frustrated magnet candidate, $\mathrm{KV}_{3} \mathrm{Sb}_{5}$. Sci. Adv. 6, eabb6003 (2020).

28. Okubo, T., Chung, S. \& Kawamura, H. Multiple- $q$ states and the skyrmion lattice of the triangular-lattice Heisenberg antiferromagnet under magnetic fields. Phys. Rev. Lett. 108, 017206 (2012).

29. Grytsiuk, S. et al. Topological-chiral magnetic interactions driven by emergent orbital magnetism. Nat. Commun. 11, 511 (2020).
30. Taguchi, K. \& Tatara, G. Anomalous Hall conductivity due to vector spin chirality in the weak coupling regime. Phys. Rev. B 79, 054423 (2009).

31. Lux, F. R. et al. Chiral Hall effect in noncollinear magnets from a cyclic cohomology approach. Phys. Rev. Lett. 124, 096602 (2020).

32. Ishizuka, H. \& Nagaosa, N. Noncommutative quantum mechanics and skew scattering in ferromagnetic metals. Phys. Rev. B 96, 165202 (2017).

33. Liang, $T$. et al. Ultrahigh mobility and giant magnetoresistance in the Dirac semimetal $\mathrm{Cd}_{3} \mathrm{As}_{2}$. Nat. Mater. 14, 280-284 (2015).

34. Ramirez, A. P. Strongly geometrically frustrated magnets. Ann. Rev. Mater. Sci. 24, 453-480 (1994).

35. Hellman, F. Interface-induced phenomena in magnetism. Rev. Mod. Phys. 89, 025006 (2017)

36. Trabel, M. et al. Twin domains in epitaxial thin MnSi layers on $\mathrm{Si}(111)$. J. Appl. Phys. 121, 245310 (2017).

37. Morikawa, D. et al. Determination of crystallographic chirality of MnSi thin film grown on $\mathrm{Si}(111)$ substrate. Phys. Rev. Mater. 4, 014407 (2020).

38. Nakatsuji, S., Kiyohara, N. \& Higo, T. Large anomalous Hall effect in a noncollinear antiferromagnet at room temperature. Nature 527, 212 (2015).

39. Manyala, N. et al. Large anomalous Hall effect in a silicon-based magnetic semiconductor. Nat. Mater. 3, 255 (2004)

40. Takahashi, K. S. et al. Anomalous Hall effect derived from multiple Weyl nodes in high-mobility $\mathrm{EuTiO}_{3}$ films. Sci. Adv. 4, eaar7880 (2018).

\section{Acknowledgements}

The authors thank A. Kitaori and M. Mogi for experimental supports and fruitful discussions. This research was supported in part by JSPS KAKENHI (Grants No. JP18J20959, No. JP20H05155, No. JP20H01859, and No. JP20H01867) and JST CREST (Grants No. JPMJCR16F1 and No. JPMJCR1874).

\section{Author contributions}

Y.T. and N.K. conceived the project. Transport and magnetization measurements using PPMS/MPMS were performed by Y.F. and N.K. High-magnetic-field transport measurements were performed by Y.F., N.K., R.K., and M.T. Thin films were grown by N.K and T.H. Theoretical calculations were provided by H.I. and N.N. Dark-field TEM measurements were performed by F.S.Y. and X.Z.Y.; Y.F., N.K., and Y.T. wrote the paper with the support from H.I., A.T., M.I., M.K., N.N., and M.T.

\section{Competing interests}

The authors declare no competing interests.

\section{Additional information}

Supplementary information is available for this paper at https://doi.org/10.1038/s41467 020-20384-w.

Correspondence and requests for materials should be addressed to Y.F., N.K. or Y.T.

Peer review information Nature Communications thanks the anonymous reviewers for their contribution to the peer review of this work.

Reprints and permission information is available at http://www.nature.com/reprints

Publisher's note Springer Nature remains neutral with regard to jurisdictional claims in published maps and institutional affiliations.

Open Access This article is licensed under a Creative Common Attribution 4.0 International License, which permits use, sharing, adaptation, distribution and reproduction in any medium or format, as long as you give appropriate credit to the original author(s) and the source, provide a link to the Creative Commons license, and indicate if changes were made. The images or other third party material in this article are included in the article's Creative Commons license, unless indicated otherwise in a credit line to the material. If material is not included in the article's Creative Commons license and your intended use is not permitted by statutory regulation or exceeds the permitted use, you will need to obtain permission directly from the copyright holder. To view a copy of this license, visit http://creativecommons.org/ licenses/by/4.0/.

(C) The Author(s) 2021 